\title{
New species and combinations on Fragilariforma (Bacillariophyta) from tropical freshwater environments
}

\author{
Pryscilla D. Almeida ${ }^{1,2^{*}}$, Carlos E. WetzeL ${ }^{3}$, Eduardo A. Morales ${ }^{4}$, Luc Ector $^{3} \&$ \\ Denise C. BICUdO ${ }^{1}$
}

\author{
${ }^{1}$ Instituto de Botânica de São Paulo, Department of Ecology, Av. Miguel Stefano 3687, 04301-902, São Paulo, \\ SP, Brazil; *Corresponding author e-mail: pryalmeida@gmail.com \\ ${ }^{2}$ Programa de Capacitação Institucional, Museu Paraense Emílio Goeldi, Campus de Pesquisa, Coord. Ciên- \\ cias da Terra e Ecologia, Av. Perimetral, 1901, Terra Firme, 66077-830, Belém, PA, Brasil \\ ${ }^{3}$ Luxembourg Institute of Science and Technology (LIST), Environmental Research and Innovation Department \\ (ERIN), 41 rue du Brill, L-4422 Belvaux, Luxembourg \\ ${ }^{4}$ Independent Consultant. Calle Andrés Uzeda 358, Colcapirhua, Cochabamba, Bolivia
}

\begin{abstract}
Several species currently belonging to the genus Fragilariforma D.M. WiLLIAMs et Round are known worldwide. This genus is mainly characterized by regular spacing of the uniseriate striae composed of round areolae bearing simple vela, and simple apical pore fields. A large number of species has been recognised for some regions of South America and tropical Africa, but few studies encompass type material and/or the morphological variation existing within populations of the different species. The aim of this study is to identify and describe the morphology of these species from tropical environments under light and scanning electron microscopy and using available type material. Nomenclatural issues are examined and clarified for five taxa: Fragilaria nitzschioides var. brasiliensis Grunow, F. undata var. brasiliensis C. ZIMmermann, F. strangulata (Zanon) D.M. Williams et Round, F. telum J.R. Carter et Denny and $F$. rolandschmidtii Metzeltin et LangeBertalot. The type material of $F$. nitzschioides var. brasiliensis, housed in the Grunow Collection at the Naturhistorisches Museum Wien (W) was examined and its epitypification is proposed. One new species from the Amazon region Fragilariforma amazonica sp. nov. is presented and described here, while Fragilaria telum is formally transferred to the genus Fragilariforma.
\end{abstract}

Key words: Africa, Brazil, diatoms, Grunow Collection, morphology, taxonomy, type material, ultrastructure

\section{INTRODUCTION}

The genus Fragilariforma was erected to include Fragilariforma virescens (RALFs) D.M. WiLliams et Round and related species. Initially named Neofragilaria D.M. Williams et Round, this name was deemed a superfluous homonym, triggering the recombination of species under the new name Fragilariforma (WILLIAMS \& Round 1987, 1988). This genus comprises several species that are distinguished from Fragilaria sensu stricto by the thin or absent sternum; larger, wider valves; higher number of copulae composing the cingulum; usually acute spines, not always linking contiguous valves; uniseriate striae composed mostly of rounded areolae bearing simple vela; and simple apical pore fields (Williams 1986, 1996; Williams \& Round 1987; Kilroy et al. 2003, Morales et al. 2012;
WeTZEL et al. 2013).

According to Williams (1996), the striae run from the sternum to the valve mantle, and the spines are tubular and located at the junction of the valve face/ mantle. Under scanning electron microscopy (SEM), the apical pore fields are present at both apices and are composed of several rows of small and round poroids. The girdle bands are open, ligulate, and bear a single row of round areolae. One rimoportula is located along a stria at one of the valve apices (WILLIAMS \& Round 1987; Kilroy et al. 2003; Morales et al. 2012).

At the time the genus was proposed, six species were transferred from Fragilaria by Williams \& Round (1988). Since then, several others have been transferred, and some others described as new (KILROY et al. 2003; Wetzel et al. 2013; Williams \& BuczKó 2016). However, the literature still contains taxa within Fragilaria that need to be transferred to Fragilarifor- 
$m a$, such is the case of $F$. nitzschioides var. brasiliensis Grunow and F. telum J.R. CARTER et DenNy.

As a whole, Fragilariforma shows great diversity in valve outline, but it is character-poor under light microscopy (LM) with the consequent existence of species complexes (Kingston et al. 2001). Within each complex there are only subtle variations among taxa, requiring the use of sophisticated tools for their separation (KInGSTON \& PAPPAS 2009). The existence of these complexes is further complicated by inadequate original descriptions, lacking sufficient detail and studies to show variability at the population level. Moreover, size reduction due to cell division during asexual reproduction frequently produces elliptic or rhombic valve shapes that are difficult to resolve even by morphometric methods (KIngSTON \& PAPPAS 2009). All this has resulted in multiple taxonomic confusions and in a literature that is difficult to interpret. According to Williams \& BuczKó (2016), at present there are about 30 species of Fragilariforma, although some of those questionably included should perhaps be placed elsewhere.

Fragilariforma virescens, for example, is reported as a cosmopolitan species (WiLliams 2001). Despite it being the generitype, there are more than 7 synonyms for the epithet virescens and 15 additional related taxa names, in addition to the 54 infraspecific entries associated to this single diatom (VANLANDINGHAM 1971; REID et al. 1994). According to the INDEX Nominum Algarum (2015), 52 infraspecific entries are found for the epithet virescens in the database.

Certainly, these complications have immediate repercussions on the use of these diatoms as ecological indicators and in the determination of their ranges of distribution (Cox 1998, 1999; Morales et al. 2001).

Fragilariforma is reported mainly from dystrophic waters varying from slightly to strongly acidic, oligo to mesotrophic environments (RENBERG 1977; Williams 1990; Kingston et al. 2001; Kilroy et al. 2003; Morales et al. 2012; Wetzel et al. 2013).

Three taxa in particular are frequently mentioned for tropical environments, i.e. Fragilariforma javanica (Hustedt) C.E. Wetzel et al., F. strangu- lata (ZANON) D.M. WILliams et Round and Fragilaria telum J.R. CARTER et DENNY, which have been historically confused with each other (HuSTEDT 1949; WILLIAMS \& Round 1987; FerRaRi \& LUdWIG 2007; WetZeL et al. 2013), and require more ultrastructural studies for the establishment of taxonomic boundaries. In Brazil, Fragilariforma javanica, Fragilaria nitzschioides var. brasiliensis, Fragilariforma strangulata and F. virescens are usually mentioned in freshwater environments (Brassac \& Ludwig 2003; Landucci \& Ludwig 2005; Dunck et al. 2012; Wetzel et al. 2012a, b; NARdelli et al. 2014). Working in the Amazon region, Wetzel (2011) reported a high diversity of Fragilariforma, citing seven taxa for this region, including $F$. javanica, $F$. nitzschioides var. brasiliensis, $F$. rolandschmidtii Metzeltin et Lange-Bertalot and $F$. virescens f. undatiformis (Ant. Mayer) Cleve-Euler. These species share characteristics that overlap, forming a complex. Also recorded from Brazil, F. undata var. brasiliensis C. ZimMERMANN was originally described from the Northeastern Atlantic Forest in Brazil, but no other record was found in the literature. Therefore, there is a common occurrence of taxa within Fragilariforma in Brazil, especially in the Amazon Region and the Atlantic Forest.

Up until now, Fragilaria javanica and F. nitzschioides var. brasiliensis have been treated as synonyms (Krammer \& Lange-Bertalot 1991; MetzelTIN \& LANGE-Bertalot 1998), but there has been no confirmation of this synonymy based on studies of type material and cross-checking with other populations. In the present paper, we study freshwater populations from rivers in the Atlantic Forest (Paiva Castro reservoir, São Paulo Metropolitan) and the Amazon basin (Rio Negro) in Brazil and additional material from Zambia, in an attempt to clarify the identity of F. nitzschioides var. brasiliensis, including analyses of the type material from the Grunow Collection. Additional SEM images from type material of $F$. javanica are also presented. A new species, F. amazonica sp. nov., is described from the Amazon region. Discussions for all taxa in the context of available literature, several synonymizations, and new combinations are provided.

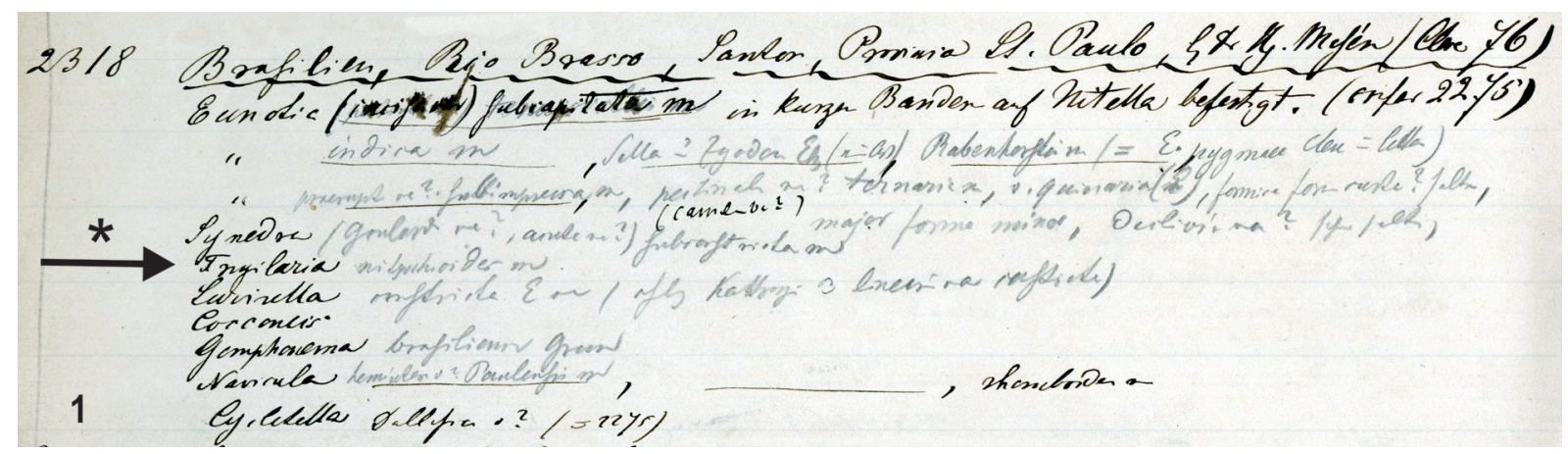

Fig. 1. Excerpt from Grunow notebook housed in the Naturhistorisches Museum Wien (W), concerning sample $n^{\circ} 2318$, with recorder of type locality Branco River, Santos, São Paulo, Brazil (i.e. "Brazilien, Rio Branco, Santos, Provincia St. Paulo"). See black arrow. 


\section{Material and Methods}

All the material used in the present manuscript is listed with additional information in Table 1. Observations are based on the original type material of Fragilaria nitzschioides var. brasiliensis from the Grunow Collection, housed in the Naturhistorisches Museum Wien (W), slide 2318. Light microscopy images were provided by Dr. Anton Igersheim, and also the drawing in plate 44, fig. 11 in VAN HeURCK (1881), including notes from Grunow's notebook on sample $\mathrm{n}^{\circ} 2318$ (Fig. 1 herein). Unfortunately, raw material sample $\mathrm{n}^{\circ} 2318$ was not available for SEM analysis. Additional SEM images of type material of $F$. javanica (also illustrated in WeTzeL et al. 2013) were obtained from the sample AS500 housed at the Hustedt Collection in Bremerhaven (BRM), Germany. Periphytic samples from Africa (Zambia) and Brazil were also studied in LM and SEM. Material from Zambia was collected scraping the surface of rocks covered with detrital material with a spoon. The collection site was located in a seep in the spray zone near the main cataract of Ntumbachushi Falls, Ngona River, Luapula Province (leg. Jonathan Taylor). Physico-chemical variables were measured concurrent with the collection of the diatom sample using a YSI 556 MPS multi parameter for temperature, electrical conductivity and oxygen concentration and a portable meter Hanna HI 98129 for $\mathrm{pH}$. More details about physical-chemistry analyses were provided by TAYLOR et al. (2014).

Samples and water chemistry data from the Brazilian Amazon were collected from several microhabitats in the Negro River basin, following procedures explained in Wetzel et al. (2012a, b). Samples from southeastern Brazil were collected from Paiva Castro Reservoir in 2013; an oligotrophic reservoir, part of the Cantareira Water Supply System, municipality Franco da Rocha, State of São Paulo. The periphytic material was collected from the natural substratum (Salvinia sp.) with a toothbrush and rinsed with distilled water. Temperature $\left({ }^{\circ} \mathrm{C}\right), \mathrm{pH}$ and conductivity $\left(\mu \mathrm{S} . \mathrm{cm}^{-1}\right)$ were measured in the field using standard electrodes (Horiba U-53). Analytical procedures for dissolved oxygen $\left(\mathrm{mg} . \mathrm{l}^{-1}\right)$, alkalinity (mEq..$\left.^{-1}\right)$, total nitrogen and total phosphorus $\left(\mu \mathrm{g} .1^{-1}\right)$ followed Standard Methods (APHA 2005).

Samples were oxidized according to methods described by the EUROPEAN COMMITTEE FOR STANDARDIZATION (2003) using concentrated $\mathrm{H}_{2} \mathrm{O}_{2}$ and $37 \%$ $\mathrm{HCl}$. Oxidized subsamples were rinsed with deionized water and permanent slides were prepared using $\mathrm{Na}$ phrax $^{\circledR}$ as mounting medium. LM images were taken at a magnification of $1000 \times$ with a Zeiss microscope Axioskop 2 equipped with DIC and phase contrast. Based on the width of the centre of the valve, apices width, width of apices at $3 \mu \mathrm{m}$ and density of striae in $10 \mu \mathrm{m}$, morphometric analysis was conducted using R program (R Core Team 2016). For SEM studies, aliquots of processed material were filtered and rinsed with deionized water through a $3 \mu \mathrm{m}$ Isopore ${ }^{\mathrm{TM}}$ polycarbonate membrane filter (Merck Millipore). Filters were mounted on aluminium stubs and coated with platinum using BAL-TEC Med 020 Modular High Vacuum Coating System for $30 \mathrm{~s}$ at $100 \mathrm{~mA}$. Cleaned 
samples were examined using an ultra-high resolution analytical field emission (FE) scanning electron microscope Hitachi SU-70 (Hitachi High Technologies) operated at $5 \mathrm{kV}$ at $10 \mathrm{~mm}$ distance. Micrographs were digitally manipulated and plates containing LM and SEM images were created using CorelDraw $\mathrm{X} 7^{\circledR}$. Morphological terminology was based on BARBER \& HAWORTH (1981) for feature of valve and Round et al. (1990) for ultrastructure and SEM terminology.

\section{Results AND Discussion}

Fragilariforma amazonica C.E. Wetzel, P.D. ALMEIDA et ECTOR sp. nov. (Figs 2-34)

Description: Valves linear, strongly constricted at the centre, marking four distinct portions; protracted apices, capitate to subcapitate in small cells (Figs 2-26). The striae are uniseriate, parallel, composed of small, circular areolae, continuous onto the mantle (Figs 27$30)$. Velum not observed. Narrow central sternum visible (Figs 2-9, 20-22, 27-30, 32). Spines present along valve edges (Figs 5, 10-13, 27-30). Apical pore fields well-developed at both apices, with several round poroids, irregularly disposed and with few spines (Figs 2-30). One rimoportula per valve near the apex, aligned with a stria (Figs 2-5, 27, 28). Spines with acutely denticulate morphology, resembling shark teeth (Figs 27-29). Girdle consisting of open bands and bearing one row of poroids (Fig. 34). Measurements from type material: length $12.0-67.0 \mu \mathrm{m}$, width at central area $2.2-5.3 \mu \mathrm{m}$, width at $1 / 3$ the valve $3.6-6.5 \mu \mathrm{m}$, width at apices 1.9-4.5 $\mu \mathrm{m}$, width at in $3 \mu \mathrm{m}$ underneath apex $1.8-4.5 \mu \mathrm{m}$, striae $18-25$ in $10 \mu \mathrm{m}(n=48)$.

Holotype: Slide SP-400392, Herbário Científico do Estado Maria Eneyda P. Kauffmann Fidalgo, holotype designated here (SP!), population partially illustrated here in LM (Figs 2-26) and SEM (Figs 27-34).

Isotype: Slide BR-4465 (Botanical Garden Meise, Belgium).

Type locality and habitat: Collected at "Igapó Ibará" a left bank tributary of the Negro River, tributary of the Amazon, State of Amazonas, Brazil. 0 ${ }^{\circ} 17^{\prime} 11^{\prime \prime}$ S, 66 35'18" W. Epilithic sample collected by C.E. Wetzel and L. Ector, sample 177 (SP-400392) on 07 March 2005.

Etymology: The specific epithet refers to the Amazon region.

Taxonomical remarks: Fragilariforma amazonica shows some similarities to $F$. constricta and $F$. javanica but differs by narrower valves and the strongly extended capitate to subcapitate apices (Table 2). In SEM, the sternum of $F$. amazonica is very narrow and often difficult to observe; spines are hollow, with ridges that are more pronounced near the base (expanding contact surface with the valve face), and disappear towards the tip of spines. The new species was found as epilithic in a tributary from the Negro River. Fragilariforma amazonica showed a few similarities with $F$. constricta for the valve outline divided in four portions and density of striae. Fragilariforma constricta has wider central area and slightly rostrate apices (PATRICK \& REIMER 1966; Zimmermann et al. 2010). SEM analyses revealed that spines found in $F$. amazonica are morphologically similar to those found in F. telum and in F. javanica, but in the latter the hollow or solid feature has not been shown. Narrow valves with extended apices and denticulate spines is a set of features only found in F. amazonica thus far (Table 2).

Fragilariforma brasiliensis (GRUnOw) P.D. AlmeIdA, C.E. Wetzel, E. Morales et D.C Bicudo comb. nov. et stat. nov. (Figs 35-70)

Basionym: Fragilaria nitzschioides var.? brasiliensis GRUNOw in Van Heurck, Synopsis des Diatomées de Belgique, pl. 44, fig. 11, 1881 .

Synonym: Fragilaria undata var. brasiliensis C. ZiMmERMANN, Broteria, Serie Botanica 17, pl. 3, fig. 7, 1919, reproduced herein Fig. 39, synon. nov.

Holotype: Slide 2318!, Grunow Collection, housed in Naturhistorisches Museum Wien (W), illustrated here in Figs 35-38.

Iconotype: Reproduced herein Fig. 35 from VAN HeURCK, Synopsis des Diatomées de Belgique, pl. 44, 1881. Epitype (designated here): Brazil, São Paulo, Paiva Castro Reservoir, Slide SP-469176 housed in Herbário Científico do Estado Maria Eneyda P. Kauffmann Fidalgo (SP). 2320'15" S, 46³9'41" W. Epiphytic sample collected by S. Zorzal and D.C. Bicudo, sample PC -03 on July 2013.

LM and SEM morphology: Frustules rectangular in girdle view (Fig. 52). Valves linear to lanceolate, constricted in the middle, subcapitate to rostrate ends (Figs 35-38 [holotype], 40-60). Some valves slightly asymmetric, small valves sometimes eccentric along the apical axis (Figs 53-60). A series of small spines is visible in LM in some valves along the valve margin (Figs 42-43). Striae uniseriate, parallel, composed of small, circular areolae, extending onto mantle, interrupted or not by spines. Each areola occluded by velum positioned near areolar outer opening (Figs 63, 68-70). One well-developed rimoportula per valve, outer opening aligned with a stria (Figs 40-46, 64, 65). Position of rimoportula in relation to valve face variable. The sternum very narrow, difficult to see even in SEM, except in valves with narrow and linear sternum (Figs 56, $61,68)$. Apical pore fields simple with several round poroids radially disposed from valve face to mantle at both valve apices. Spines sometimes present on apical pore fields, irregularly arranged or in semicircle (Figs 62, 63, 68, 69). Spines solid, conical, acutely ended, slightly curved and irregularly positioned on costae or striae (Figs 61, 68-70). Mantle deep, ornamented at abvalvar margin with plaques (Fig. 67). Cell dimensions from type and epitype material: length 10.7-74.4 


\begin{tabular}{|c|c|c|c|c|c|c|c|c|c|c|c|c|}
\hline 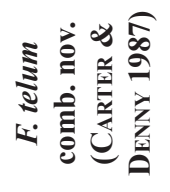 & $\infty$ & $q$ & & in & $\begin{array}{l}b \\
i n\end{array}$ & $\stackrel{r}{f}$ & $\infty$ & 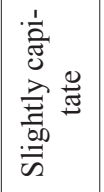 & 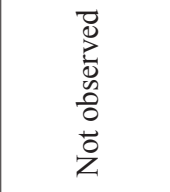 & $\begin{array}{l}\vec{D} \\
\sum_{0}^{0} \\
0 \\
0 \\
0 \\
0 \\
z\end{array}$ & 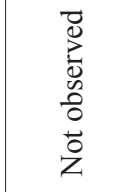 & $\begin{array}{l}\vec{D} \\
\sum_{0}^{0} \\
0 \\
0 \\
0 \\
0 \\
z\end{array}$ \\
\hline 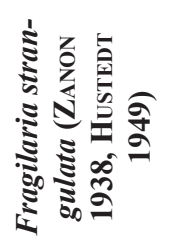 & $\begin{array}{l}\infty \\
\infty \\
\approx\end{array}$ & $\begin{array}{l}\infty \\
b\end{array}$ & $\tilde{n}$ & $\begin{array}{l}\dot{p} \\
\dot{p} \\
\dot{m}\end{array}$ & $\nabla$ & $\frac{\hat{i}}{\hat{i}}$ & $\begin{array}{l}\text { ô } \\
\text { त̃ } \\
\hat{~} \\
\text { ते }\end{array}$ & 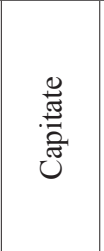 & 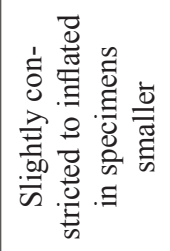 & 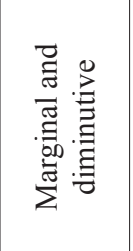 & 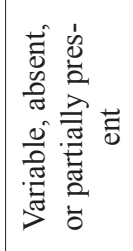 & 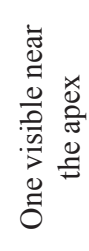 \\
\hline 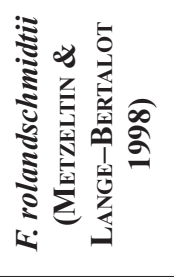 & $\begin{array}{l}\text { a } \\
\text { d }\end{array}$ & $\tilde{f}$ & 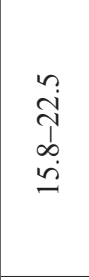 & $\begin{array}{l}n \\
\stackrel{n}{+} \\
\dot{m}\end{array}$ & $\stackrel{n}{m}$ & $\stackrel{i}{i}$ & $\begin{array}{l}\hat{i} \\
\text { iे }\end{array}$ & 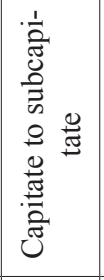 & 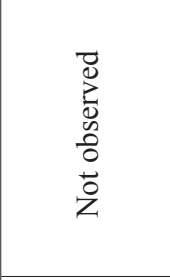 & $\begin{array}{l}\vec{D} \\
\vec{D}^{\circ} \\
0 \\
0 \\
0 \\
\overrightarrow{0}\end{array}$ & $\begin{array}{l}\vec{D} \\
\vec{D}_{0} \\
0 \\
0 \\
0 \\
\overrightarrow{0}\end{array}$ & $\begin{array}{l}\vec{B} \\
\stackrel{D}{0} \\
0 \\
0 \\
0 \\
0 \\
\overrightarrow{0}\end{array}$ \\
\hline 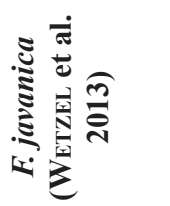 & $\begin{array}{l}n \\
i \\
\infty \\
\dot{v} \\
\dot{v}\end{array}$ & $\begin{array}{l}0 \\
\dot{\varphi} \\
i\end{array}$ & $\stackrel{\oplus}{I}$ & $\begin{array}{l}n \\
n \\
n\end{array}$ & $\frac{m}{\stackrel{m}{+}}$ & $\frac{n}{\stackrel{n}{i}}$ & $\begin{array}{l}\stackrel{\widehat{~}}{b} \\
\text { - }\end{array}$ & 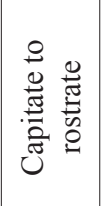 & 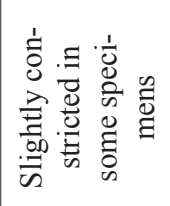 & 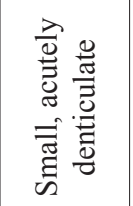 & 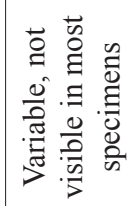 & 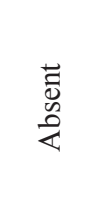 \\
\hline 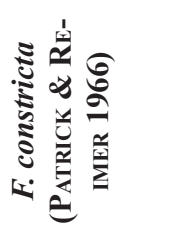 & î & $\frac{0}{b}$ & $\overleftrightarrow{\breve{z}}$ & $\overleftrightarrow{\breve{z}}$ & 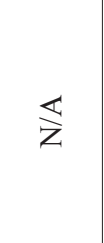 & $\overleftrightarrow{\mathrm{z}}$ & $\frac{\infty}{n}$ & 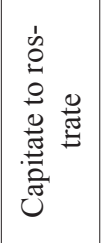 & 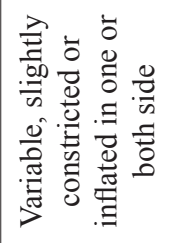 & 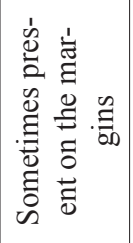 & 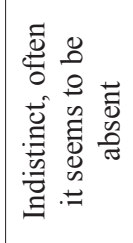 & $\begin{array}{l}\overrightarrow{0} \\
\vec{D}_{0}^{0} \\
0 \\
0 \\
0 \\
\overrightarrow{0}\end{array}$ \\
\hline 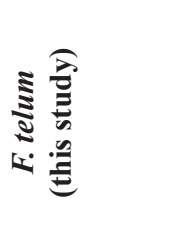 & $\stackrel{̊}{f}$ & $\begin{array}{l}n \\
\vdots \\
\dot{m} \\
\dot{n}\end{array}$ & $\stackrel{\sim}{I}$ & $\begin{array}{l}n \\
0 \\
n \\
n \\
n\end{array}$ & $\begin{array}{l}\infty \\
i \\
\stackrel{1}{I}\end{array}$ & $\begin{array}{l}\vec{t} \\
\vec{g}\end{array}$ & $\begin{array}{l}\tilde{\hat{~}} \\
\underline{-}\end{array}$ & 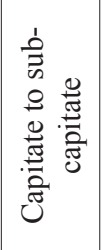 & 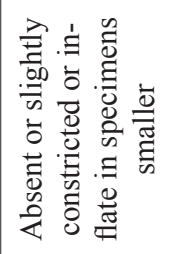 & 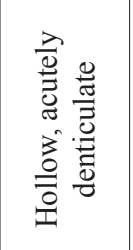 & 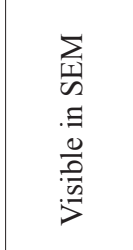 & 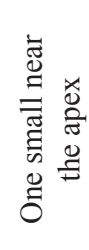 \\
\hline 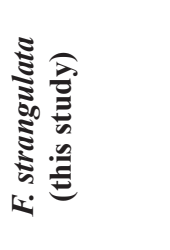 & \begin{tabular}{l} 
Na \\
\multirow{0}{0}{} \\
0 \\
\end{tabular} & $\begin{array}{l}\vec{\infty} \\
\dot{\gamma}\end{array}$ & $\underset{\stackrel{g}{+}}{\dot{j}}$ & $\begin{array}{l}\infty \\
\dot{0} \\
o \\
\dot{m}\end{array}$ & $\begin{array}{l}\infty \\
\infty \\
\infty \\
i \\
i\end{array}$ & $\begin{array}{l}\underset{+}{+} \\
\stackrel{y}{y}\end{array}$ & 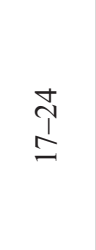 & 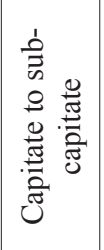 & 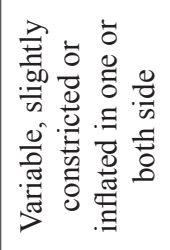 & 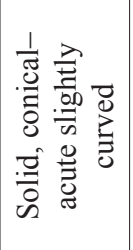 & 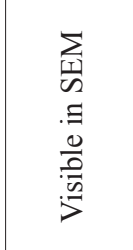 & 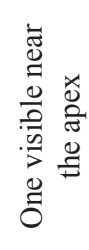 \\
\hline 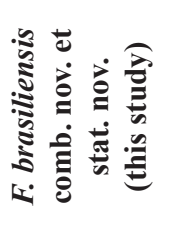 & $\begin{array}{l}\vec{j} \\
\vec{a} \\
\dot{j}\end{array}$ & $\begin{array}{l}\vec{\infty} \\
0 \\
\alpha \\
\dot{r}\end{array}$ & 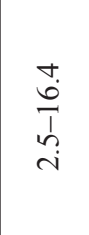 & $\begin{array}{l}\infty \\
\dot{0} \\
o \\
\text { r. }\end{array}$ & $\begin{array}{l}\infty \\
\stackrel{i}{~} \\
\stackrel{-1}{-}\end{array}$ & $\stackrel{\infty}{\stackrel{\infty}{+}}$ & $\stackrel{0}{\stackrel{1}{I}}$ & 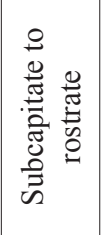 & 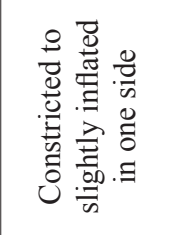 & 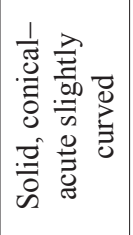 & 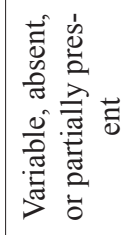 & 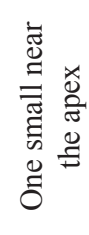 \\
\hline 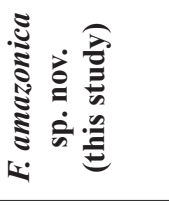 & 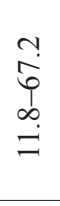 & 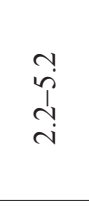 & $\begin{array}{l}\hat{\imath} \\
\hat{i} \\
i\end{array}$ & $\begin{array}{l}+ \\
b \\
b \\
\dot{m}\end{array}$ & $\begin{array}{l}n \\
\dot{+} \\
\stackrel{\infty}{-}\end{array}$ & $\begin{array}{l}\infty \\
\dot{j} \\
\dot{b}\end{array}$ & $\begin{array}{l}\hat{\imath} \\
\infty \\
\infty\end{array}$ & 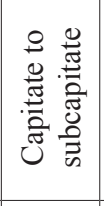 & 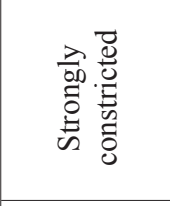 & 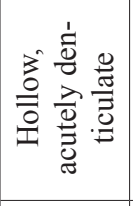 & 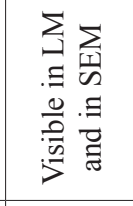 & 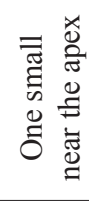 \\
\hline & 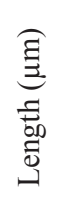 & 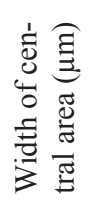 & 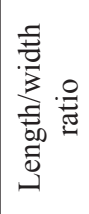 & 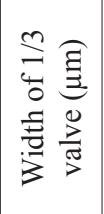 & 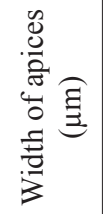 & 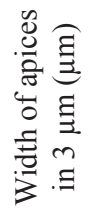 & 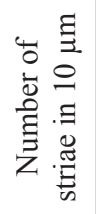 & $\begin{array}{l}0 \\
.00 \\
\frac{2}{2}\end{array}$ & 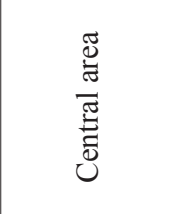 & $\begin{array}{l}\text { o } \\
\text { : } \\
\text { की }\end{array}$ & $\begin{array}{l}\text { 音 } \\
\text { 离 } \\
\text { 品 }\end{array}$ & 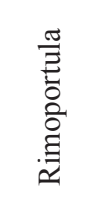 \\
\hline
\end{tabular}


$\mu \mathrm{m}$, width at central area $3.5-6.7 \mu \mathrm{m}$, width at $1 / 3$ the valve 3.6-6.5 $\mu \mathrm{m}$, width at apices 1.9-4.4 $\mu \mathrm{m}$, width at in $3 \mu \mathrm{m}$ underneath apex $1.2-4.7 \mu \mathrm{m}$, striae $19-26$ in $10 \mu \mathrm{m}(\mathrm{n}=80)$.

Taxonomical remarks: Based on LM analysis, the recent freshwater material collected in Paiva Castro Reservoir (São Paulo) is very similar to Fragilaria nitzschioides var. brasiliensis described by GRUNOw in VAN Heurck 1881 from Branco River, Santos, São Paulo (Fig. 70). The description of Fragilariforma brasiliensis presented here is supported by the drawing in VAN HEURCK and images taken from the holotype slide included here (Figs 35-38). This species differs from Fragilariforma nitzschioides (GRUNOw) LANGE-BERTALOT in Hofmann et al. (2011: 268, pl. 6, figs 9, 10) by the shape of the apices, spines, sternum, frustule shape in girdle view; therefore, it can no longer be considered as a variety (KRAMMER \& LANGE-BERTALOT 1991; Howard \& Morales 2012). The apices of F. nitzschioides are slightly rostrate to abruptly rounded, sternum is present along the apical axis, striae on either side of the sternum, short spines, of the valve and frustules arranged in ribbon-like colonies (HowARD \& MORALES 2012; Wojtal 2013). Fragilaria brasiliensis has prom- inent apices, very narrow sternum, or absent in some valves, and in this study we could not find evidence of colonies or linking spines. Thus a new nomenclatural status is required.

Fragilariforma brasiliensis was treated as a synonym of $F$. javanica (Hustedt) C.E. Wetzel, E. Morales et Ector by Krammer \& Lange-Bertalot (1991) and Metzeltin \& Lange-Bertalot (1998). The identification of $F$. brasiliensis in Brazil was based on these references and, therefore, F. brasiliensis was mistaken as $F$. javanica in different regions of the country (Metzeltin \& Lange-Bertalot 1998; Brassac \& LudWIG 2003; LANDUCCI \& LudWIG 2005; FERRARI \& LuDwig 2007; WetZel 2011; Dunck et al. 2012; NARdelli et al. 2014). We show, by means of LM and SEM information that these species differ in form and width of apices and kind of spines (Figs 62-70). In the type material, $F$. javanica valves did not present rimoportula (WETzel et al. 2013, see figs 18-20), new photos obtained from this type material, revealed a slender septum (Figs 71, 72), although in the material from Brazil the valves did not present this structure, thus indicating that populations studied here belong to $F$. brasiliensis. To clarify the separation of $F$. brasiliensis and $F$. javanica, a comparison between both taxa under SEM
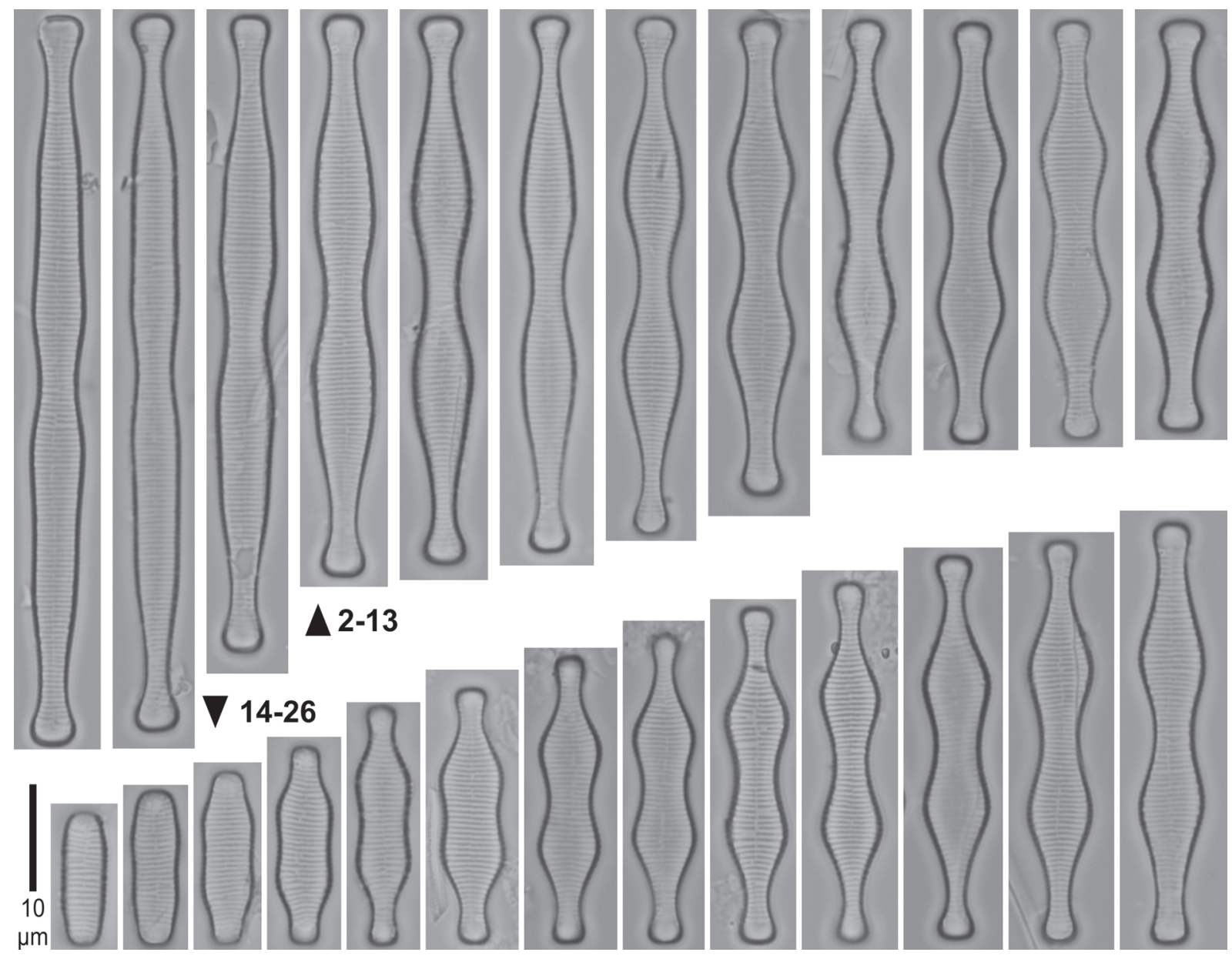

Figs 2-26. LM images of Fragilariforma amazonica sp. nov. C.E. WetzeL, P.D. AlmeIDA et Ector from the type material (SP400392). Specimens showing size diminution series. Scale bar $10 \mu \mathrm{m}$. 
was necessary. Unfortunately, there was no raw material of F. brasiliensis at the Grunow Collection for SEM, consequently, an epitype from Paiva Castro reservoir (São Paulo) was selected to facilitate interpretation, as recommended by the International Code of Nomenclature for algae, fungi, and plants (REID et al. 1994; JAHN \& Kusber 2009; McNeILl et al. 2012). The designation of an epitype was necessary given the importance of unmounted material for SEM analysis. The type material present in the Grunow Collection (W) consists only of a permanent slide, and it is not possible to use it for the ultrastructural analyzes that are required.

This reservoir was selected due to the large population found developing in the periphyton (on Salvinia sp.),
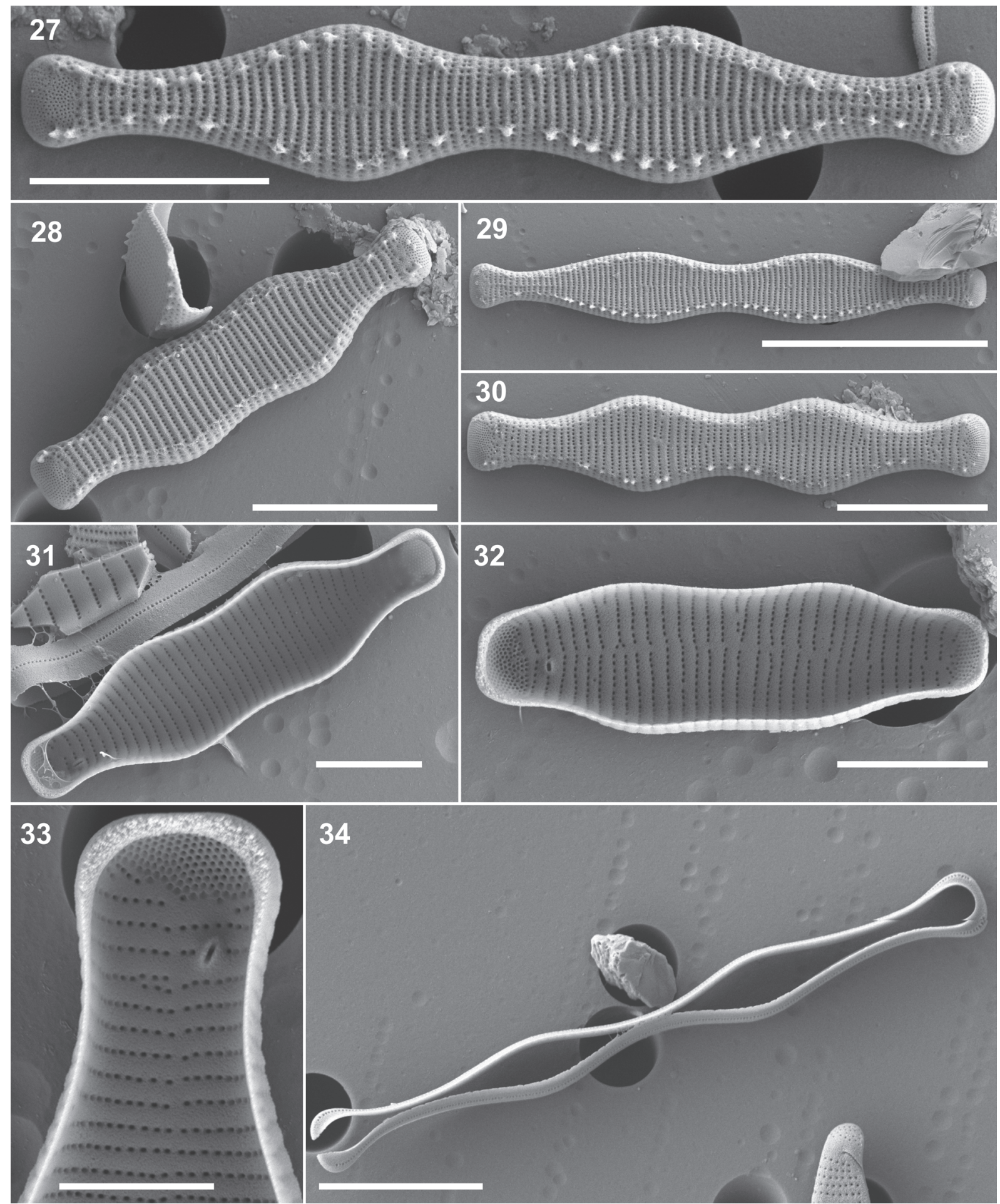

Figs 27-34. SEM images of Fragilariforma amazonica sp. nov. C.E. Wetzel, P.D. AlmeIDA et Ector from the type material (SP400392): (27-30) valves in external view; (31-32) valves in internal view; (33) apex with rimoportula in internal view; (34) girdle band open with ornamentation. Scale bar $20 \mu \mathrm{m}(29), 10 \mu \mathrm{m}(27,28,30,34), 5 \mu \mathrm{m}(31,32), 3 \mu \mathrm{m}(33)$. 
but also because the type locality of $F$. brasiliensis, Branco River, Santos, São Paulo disappeared due to urban developement.

Fragilariforma undata var. brasiliensis C. ZIMMERMANN was described from the State of Bahia, northeastern Brazil. This taxon differed from $F$. brasiliensis by its inconspicuous sternum and absence of spines according to ZimmermanN (1919). However, these characters are not sufficient for consistent differentiation of taxa according to the revision of the genus by WILLIAMS \& Round (1987), where the authors state that in taxa within Fragilariforma spines may not be observed in some specimens (Figs 39, 44-46) and the sternum is variable (Williams 1996; Kilroy et al. 2003; Morales et al. 2012). Therefore, the synonymization of $F$. undata var. brasiliensis with $F$. brasiliensis is proposed since both taxa clearly coincide in shape variability (valve outline) and morphometric measures (see Figs 35-60). The synonymization of $F$. brasiliensis with $F$. undata var. brasiliensis is justified based on the lack of a valid diagnostic character of this variety. Unfortunately, the fate of Zimmermann's collection is unknown, and any slides used by Zimmermann in his studies are unavailable (Gomes et al. 2012).

\section{Fragilariforma strangulata (ZANON) D.M. WiLliams} et Round (Figs 73-94)

Basionym: Synedra strangulata ZANON, Commentationes, Pontificia Academia Scientiarum, p. 587, fig. 14, 1938.

Material analysed: Zambia, Ntumbachushi Falls, Ngona River, Luapula Province, sample CCA-1237, $\mathrm{BR}-4375$ (BR!).

LM and SEM morphology: Frustules rectangular in girdle view (Figs 83, 84). Valves linear to widely lanceolate, slightly concave valvar sides in larger specimens, gibbous outline in smaller ones (Figs 78, 8082). Ends capitate to subcapitate (Figs 73-82). Striae uniseriate, parallel, composed of small, circular areolae. Striae extending from valve face to mantle (Figs 85-87). Velum not observed. Narrow sternum, absent in some valves (Fig. 85). Apical pore fields well-developed at both apices, with several round poroids, irregularly disposed; sometimes with spines (Figs 88-91).

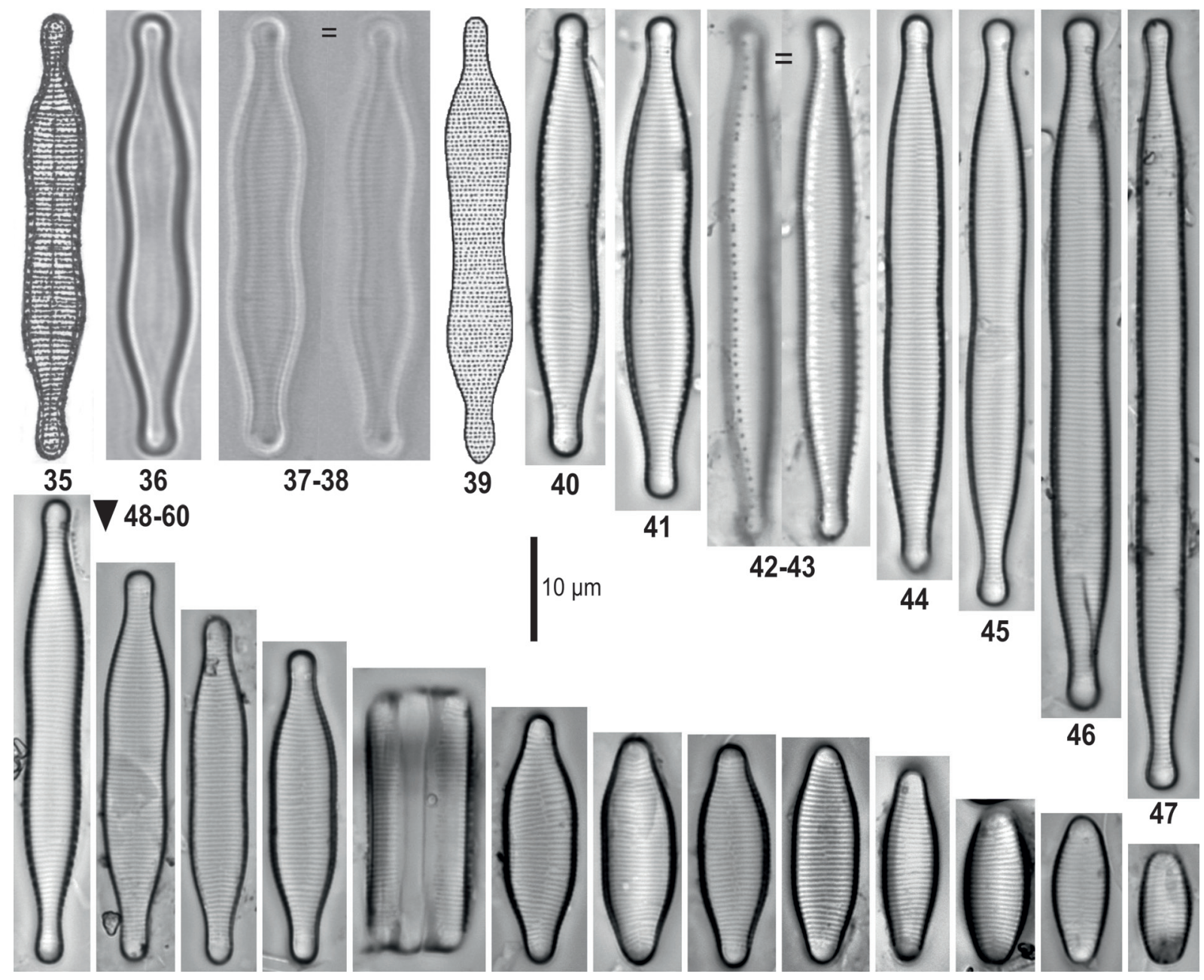

Figs 35-60. Images of Fragilariforma brasiliensis (Grunow) P.D. Almeida, C.E. Wetzel, E. Morales et D.C. Bicudo: (35-38) images from type material. 1 Iconotype; (36-38) LM images from type material (slide 2318); (39) iconotype of Fragilaria undata var. brasiliensis; (40-60) LM images of population from epitype locality Paiva Castro reservoir (SP469176). Scale bar $10 \mu \mathrm{m}$. 
Spines solid, conical-acute slightly curved, irregularly positioned (Figs 93, 94). One rimoportula per valve near the apex, located along a stria, internally welldeveloped (Figs 81, 82, 85, 86, 89, 91). Mantle wide, ornamented with irregularly spaced silica plaques (Fig. 87). Girdle consists of up to eight open bands and bearing one row of poroids (Fig. 87). Cell dimensions measured in material from Zambia, Austral Africa: length 26.0-64.0 $\mu \mathrm{m}$, width at central area 4.0-8.1 $\mu \mathrm{m}$, width at $1 / 3$ the valve $3.9-6.8 \mu \mathrm{m}$, width at apices $2.8-3.8$ $\mu \mathrm{m}$, width at in $3 \mu \mathrm{m}$ underneath apex 2.2-4.7 $\mu \mathrm{m}$, striae $17-24$ in $10 \mu \mathrm{m}(\mathrm{n}=36)$.

Taxonomical remarks: The population analyzed from Zambia, identified as F. strangulata, is consistent with descriptions and illustrations presented in ZANON (1938) and HustedT (1949). This taxon was originally found in material from Africa and Ecuador by ZANON, who selected samples from Congo as the type of what he called Synedra strangulata. In the same paper ( $\mathrm{Z}_{\mathrm{A}-}$ NON 1938), one new variety was described, Synedra famelica var. enflata ZANON also from Congo. This variety was posteriorly placed within a continuum of the $F$. strangulata variability by HustedT (1949). In the latter concept, $F$. strangulata includes extremely variable morphologies, the smaller valves are distended transapically, while longer valves tend to be linear, spines and narrow sternum are not constant, and there is one rimoportula near the apex (HustedT 1949). Fragilariforma strangulata is closely related with $F$. javanica, due to similar shape of valves and presence of spines (WILliams \& Round 1987). In this study, the comparison between the type material of $F$. javanica (Table 2) and analyses of populations of F. strangulata evidenced differences in LM and SEM such as presence of rimoportula, shape and width of apices, spines change, sternum, and velum confirming the distinction between these two taxa (see also WeTzel et al. 2013).

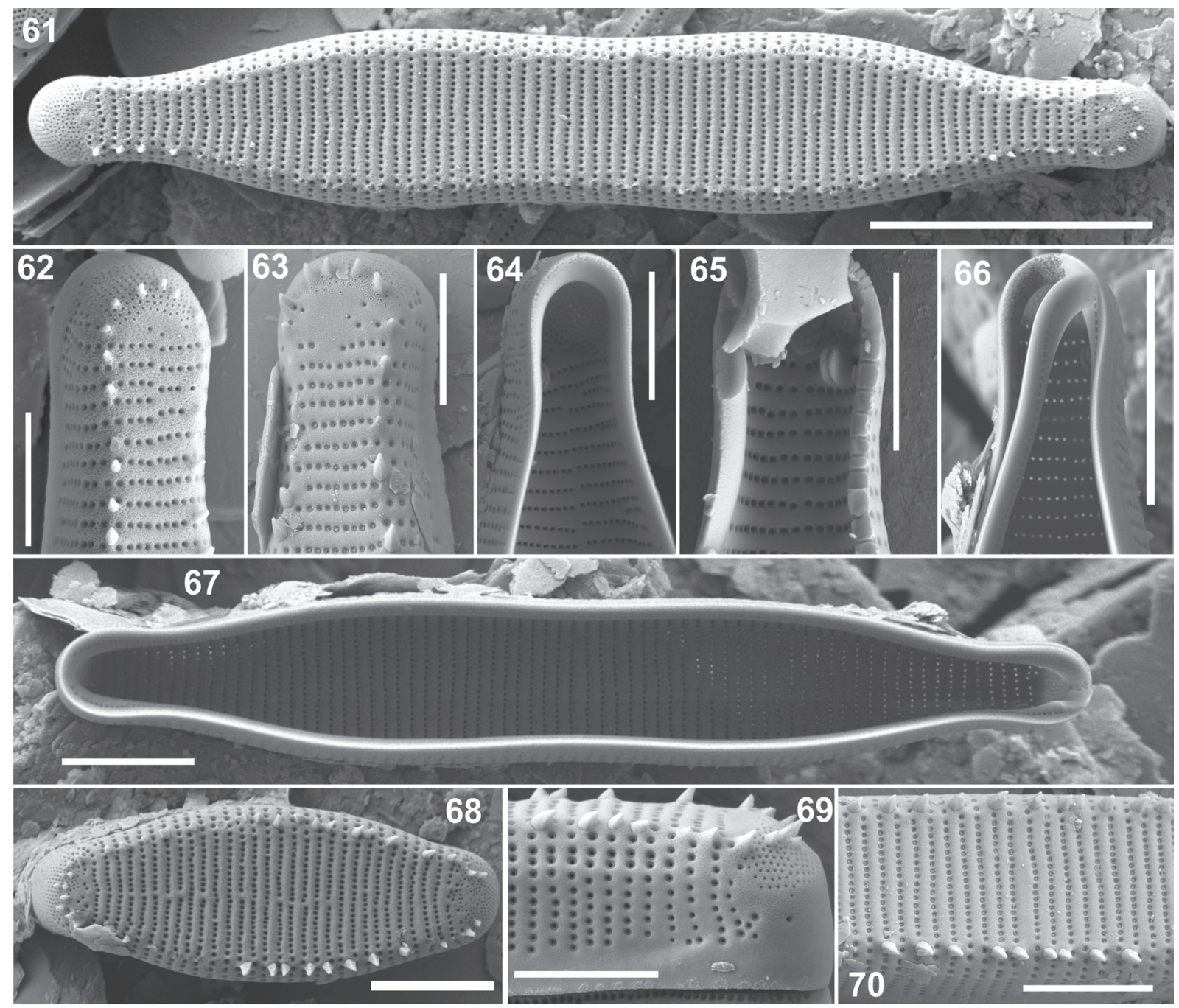

Figs 61-70. SEM images of Fragilariforma brasiliensis (Grunow) P.D. Almeida, C.E. Wetzel, E. Morales et D.C. Bicudo from epitype locality Paiva Castro reservoir (SP469176): (61, 68) valves in external view; (62-63) apices in external view without rimoportula; (64-66) apices in internal view; (64-65) apices with rimoportula; (67) valve in internal view; (69) apex in girdle view showing curved spines; (70) central area in external view with conical-acute spines. Scale bar $10 \mu \mathrm{m}(61,67), 4 \mu \mathrm{m}(64,66,70), 3 \mu \mathrm{m}(62,63,65,68,69)$. 


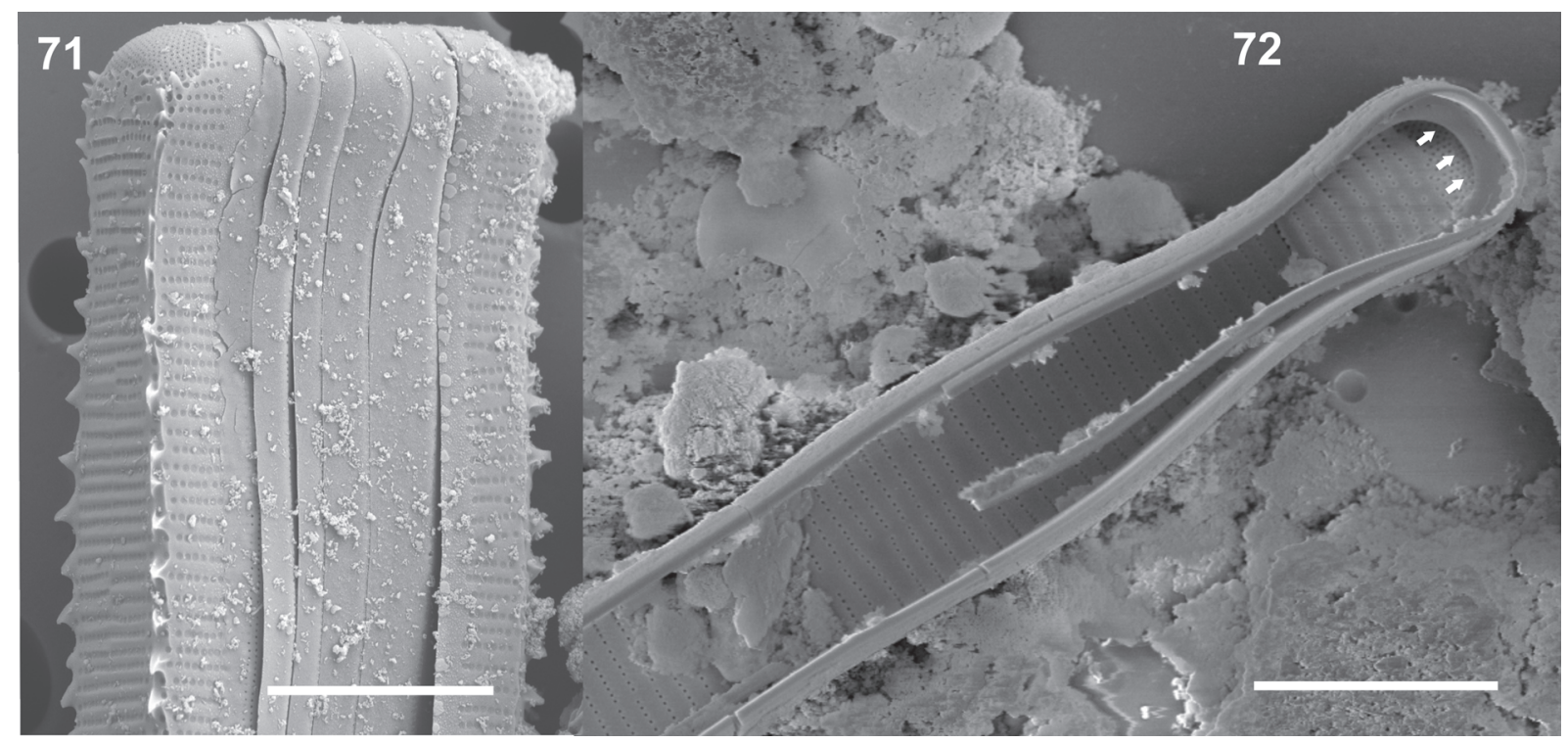

Figs 71, 72. SEM images from type material (BRM-AS500) of Fragilariforma javanica C.E. WetZEL, E. MoRALES et EctoR and septum: (71) frustule in girdle view showing denticulate spines; (72) valve in internal view showing septum (white arrow). Scale bars $10 \mu \mathrm{m}$.
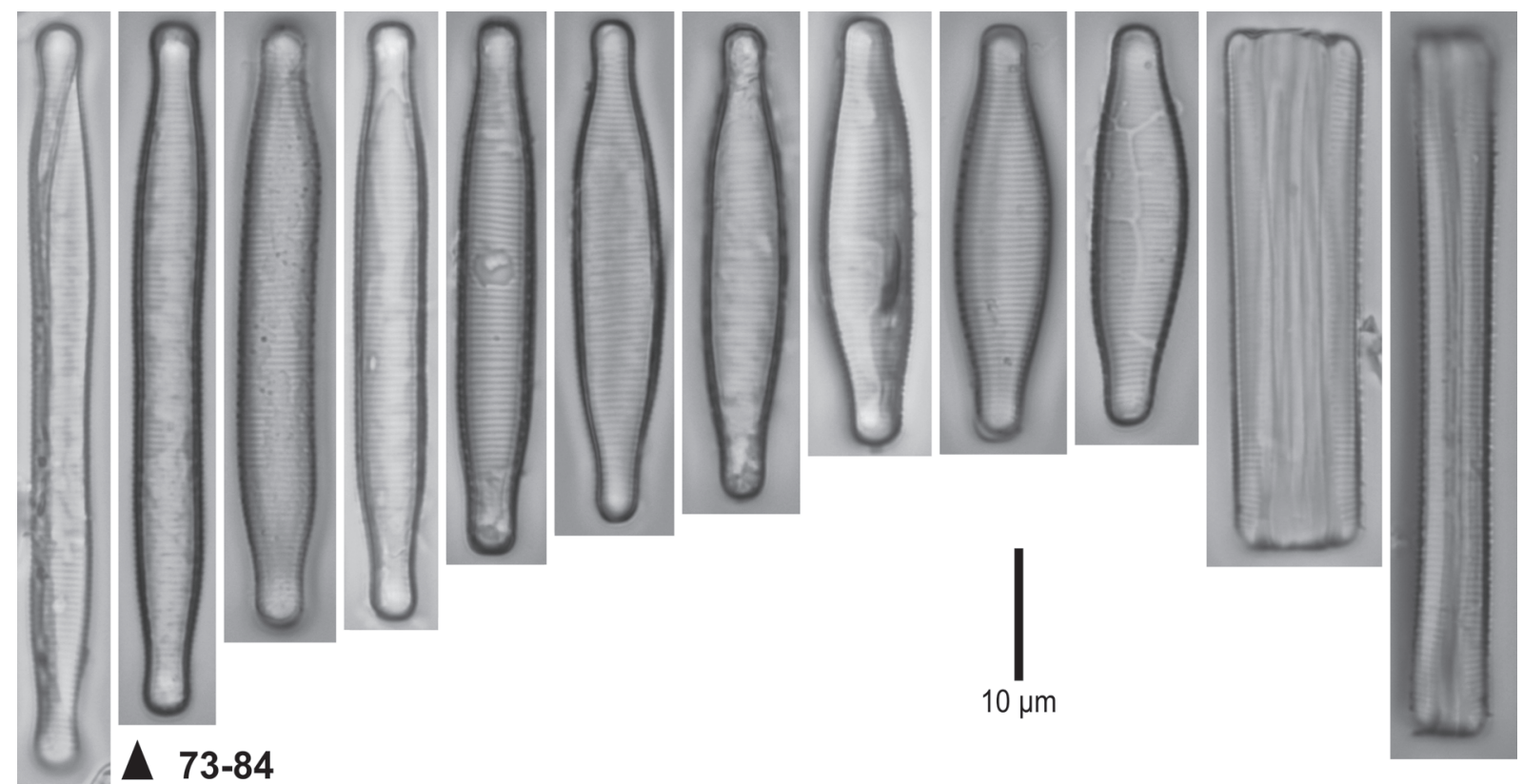

Figs 73-84. LM images of Fragilariforma strangulata (ZANON) D.M. Williams et Round from Ntumbachushi Falls, Zambia (CCA-1237). Scale bar $10 \mu \mathrm{m}$.

Fragilariforma telum (J.R. CARTER et DenNy) P.D. Almeida, C.E. Wetzel et E. Morales comb. nov. (Figs 95-129)

Basionym: Fragilaria telum J.R. CARTER et DENNY, Nova Hedwigia 44, pl. 11, fig. 25, 1987.

Synonym: Fragilaria rolandschmidtii Metzeltin et Lange-BertaLOT, Iconographia Diatomologica 5, pl. 1, figs 14-16, 1998, synon. nov.

Material analysed: Brazil, Negro River, Santa Isabel, sample RN65, SP400280.

LM and SEM morphology: Valves linear to narrow elliptic, with slightly median constriction, capitate to subcapitate apices (Figs 95-106). Small cells with projection along apical axis (Figs 107-117). Striae uniseriate, parallel, composed of small, circular areolae. Striae extending from valve face to mantle (Figs 120-125). Velum not observed. Sternum variable, difficult to visualize in LM, absent (Fig. 126) or restricted to distal portion in some valves (Fig. 118). Apical pore fields of different sizes, composed of several round poroids irregularly disposed on valve mantle and bearing few spines (Figs 120-123). Small spines very difficult to visualize (Figs 106, 110). Spines hollow, acutely denticulate, resembling shark teeth (Figs 118, 124, 

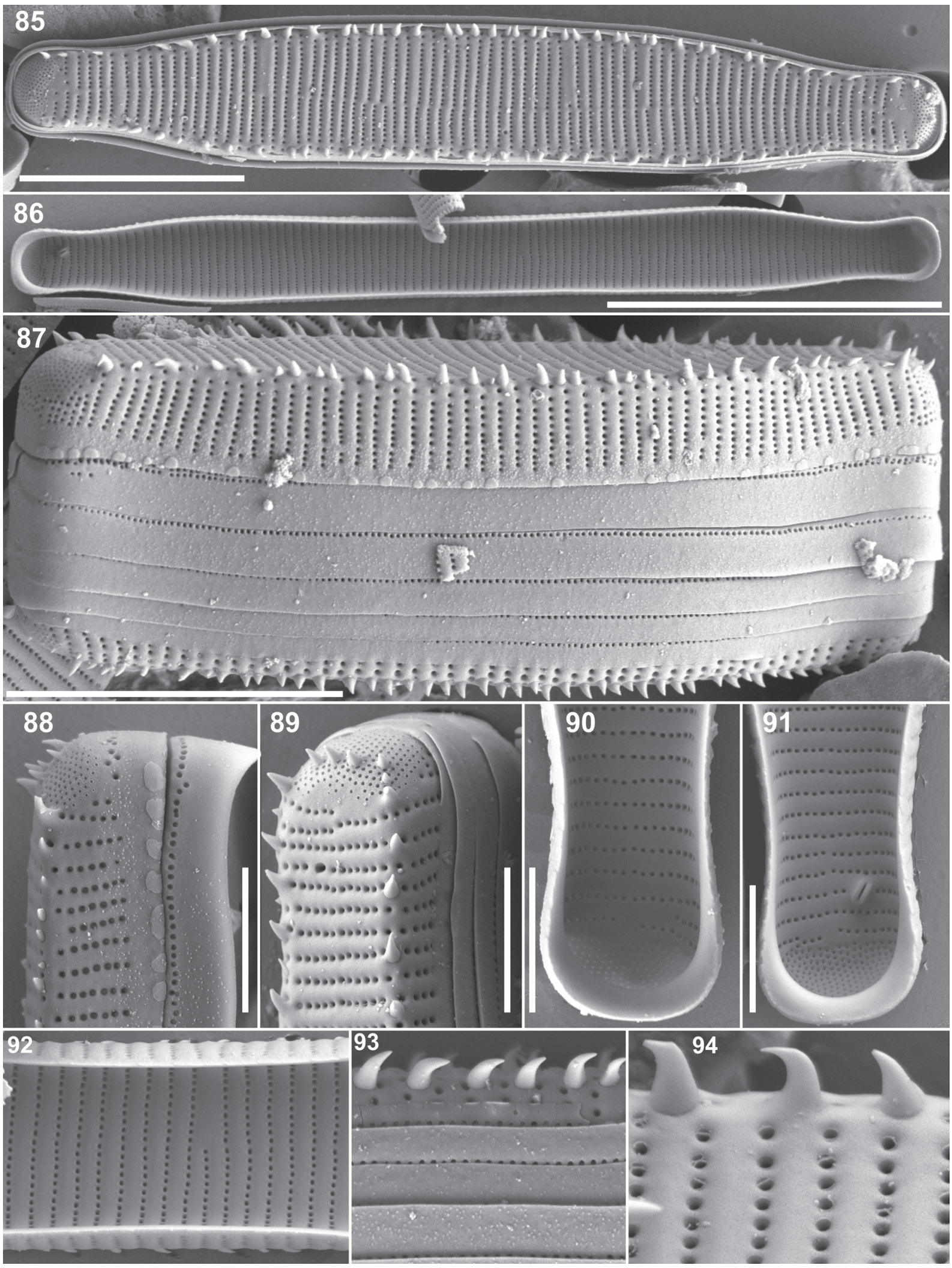

Figs 85-94. SEM images of Fragilariforma strangulata (ZANon) D.M. WiLLiams et Round from Ntumbachushi Falls, Zambia (CCA-1237): (85) valve in external view; (86) valve in internal view; (87) frustule in girdle view showing several spines; (88) apex in girdle view showing spines and numerous silica plaques on mantle; (89) apex in external view with rimoportula; $(90,91)$ apices in internal view, $(91)$ apex with rimoportula; (92) central area in internal view with one shortened stria; (93) frustule in girdle view showing spines; (94) central area with curved spines. Scale bar $20 \mu \mathrm{m}$ (86), $10 \mu \mathrm{m}$ (85, 87), $3 \mu \mathrm{m}(88-91), 2 \mu \mathrm{m}$ (92, 93), $1 \mu \mathrm{m}$ (94). 
126). One rimoportula is aligned with a stria, internally well-developed (Figs 120, 122, 128). Cell dimensions measured in population from Negro River in Brazilian Amazon: length 4.0-100.0 $\mu \mathrm{m}$, width at central area $3.7-6.3 \mu \mathrm{m}$, width at $1 / 3$ the valve $3.5-6.5 \mu \mathrm{m}$, width at apices 1.9-5.8 $\mu \mathrm{m}$, width at in $3 \mu \mathrm{m}$ underneath apex $2.1-4.0 \mu \mathrm{m}$, striae $16-22$ in $10 \mu \mathrm{m}(\mathrm{n}=70)$.

Taxonomical remarks: We identified a population obtained from Negro River as F. telum following illustrations presented in CARTER \& Denny (1987). The type material of $F$. telum was described from Sierra Leone (Africa) with diagnosis very closely related to $F$. javanica, but it differs in the morphology of spines and always absent sternum. These two characters are very difficult to observe in LM and were not illustrated in CARTER \& DENNY (1987). Another taxon very similar is $F$. rolandschmidtii described from the Amazon region by Metzeltin \& Lange-Bertalot (1998). Since both taxa are similar concerning their diagnoses, including valve shape, length and width ranges, and density of striae in $10 \mu \mathrm{m}$ (Table 2 ), there are no exclusive characters to separate these taxa and so should be treated as one, possessing a wide distribution in tropical environments and are considered by us as synonyms.

Our results in SEM showed presence of small marginal spines in F. telum. The spine shapes are similar to those found in $F$. javanica (Wetzel et al. 2013), nevertheless in F. telum spines are hollow, smaller and irregularly distributed in the margins of valves.
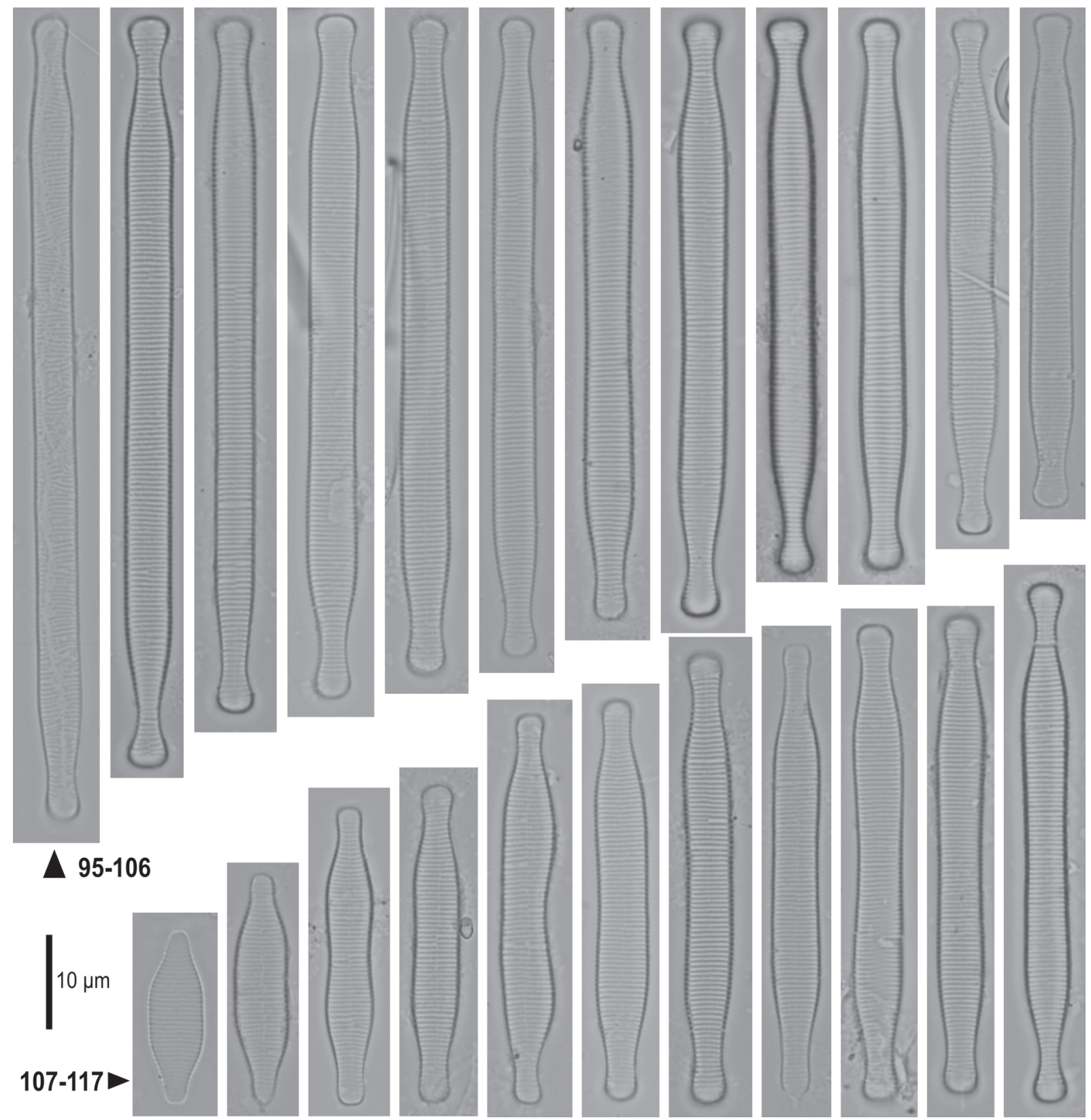

Figs 95-117. LM images of Fragilariforma telum (J.R. Carter et Denny) P.D. Almeida, C.E. Wetzel et E. Morales from Negro River (SP400280), Amazon, Brazil. Scale bar $10 \mu \mathrm{m}$. 
Recently, Alfinito \& Lange-Bertalot (2013) studied diatoms from Sierra Leone and identified a taxon without spines as $F$. javanica. These authors emphasize the similarities between $F$. telum and $F$. javanica and the necessity to compare them under SEM to under- stand their relatedness. Fragilaria javanica HustedT was transferred to the genus Fragilariforma based on detailed analysis of type material using LM, SEM, ecological and distribution data (WeTZEL et al. 2013). This taxon is commonly reported from tropical and
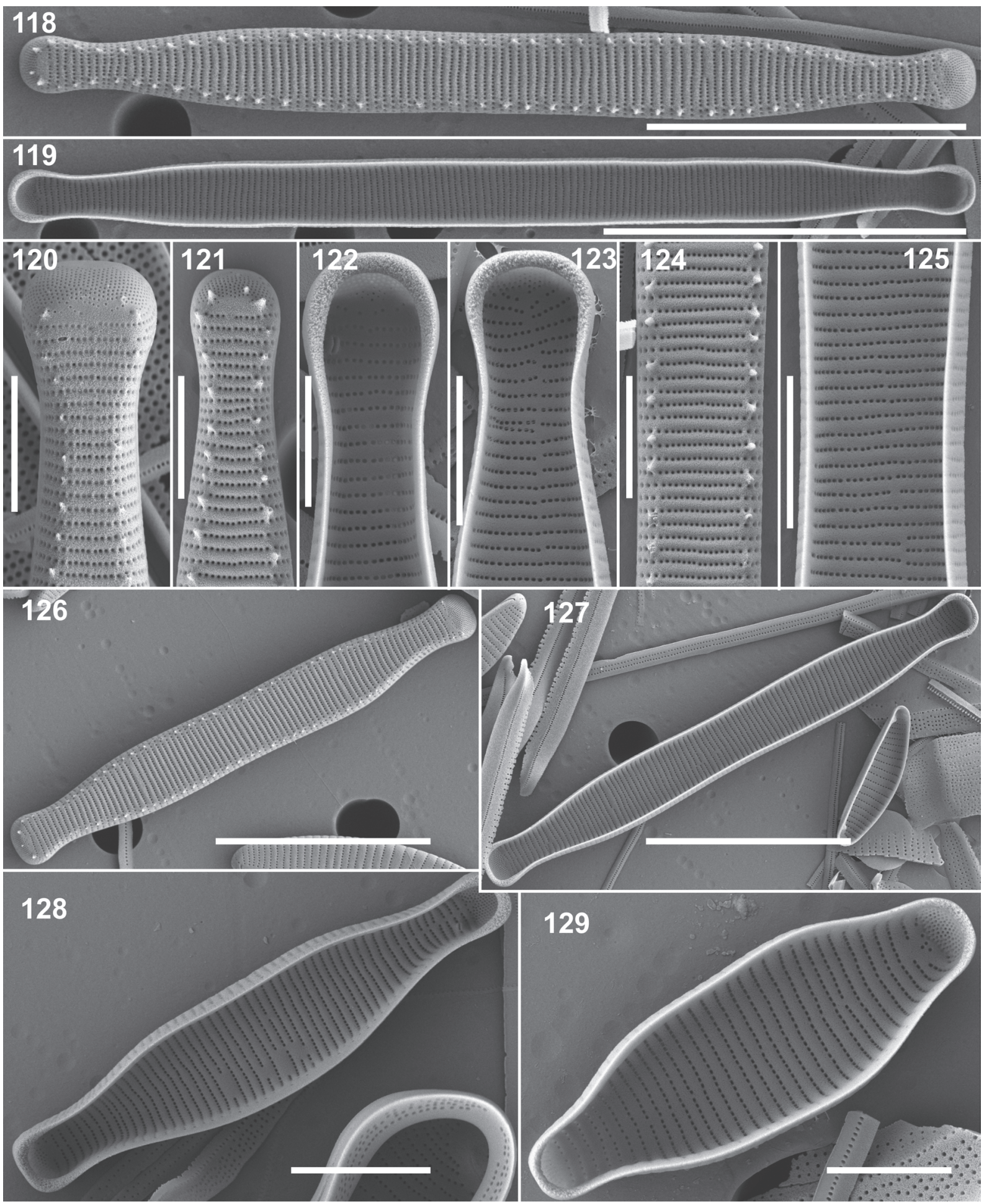

Figs 118-129. SEM images of Fragilariforma telum (J.R. Carter et Denny) P.D. Almeida, C.E. Wetzel et E. Morales from Negro River (SP400280), Amazon, Brazil: $(118,126)$ valves in external view; $(119,127-129)$ valves in internal view; $(120-121)$ apices in external view, (120) apex with rimoportula; (122-123) apices in external view showing narrow sternum, (122) apex with rimoportula; (124) central area in external view showing denticulate spines; (125) central area in internal view. Scale bar $30 \mu \mathrm{m}(119), 20 \mu \mathrm{m}(118,126,127), 10 \mu \mathrm{m}(128), 5$ $\mu \mathrm{m}(120,121,123-125,129), 4 \mu \mathrm{m}(122)$. 

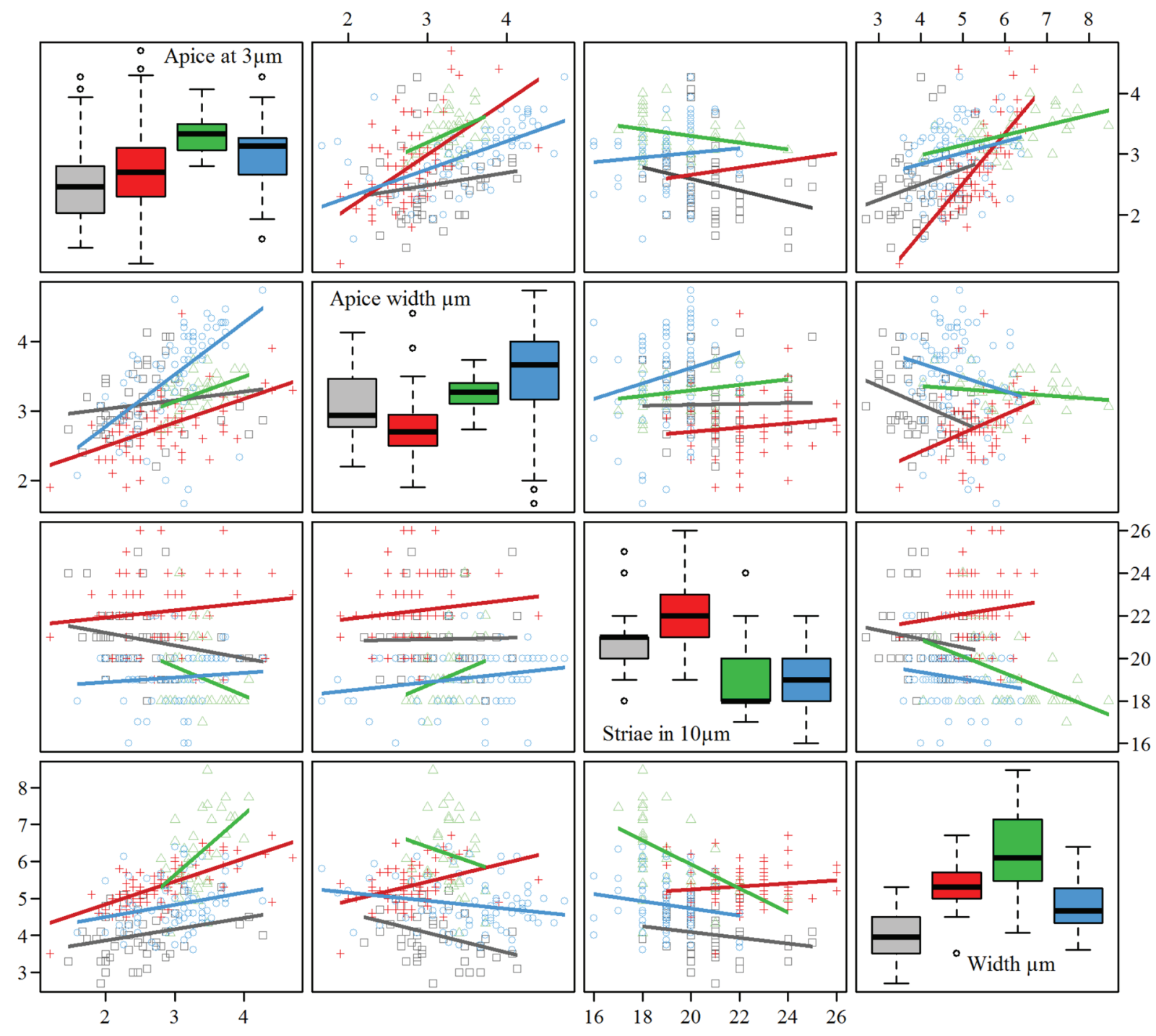

$\square$ F. amazonica
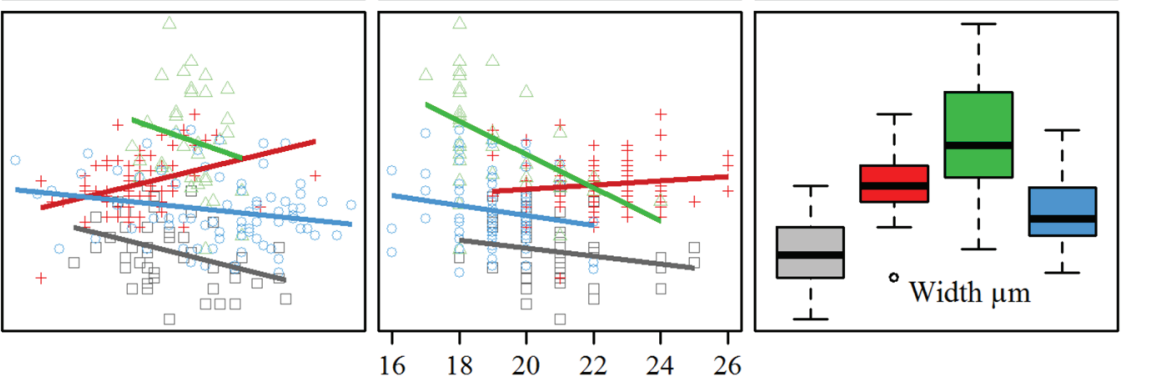

Fig. 130. Morphometric data for separation between Fragilariforma species from tropical environments.

subtropical areas (as subfossil) and it is closely related to taxa currently associated with $F$. virescens (WETZEL et al. 2013). As in F. javanica, the regular absence of rimoportula could also be observed (see Fig. 129). In this study, we transfer Fragilaria telum J.R. CARTER et DenNy into Fragilariforma.

In summary, the ultrastructural analysis of the type material from Brazil was necessary to partially solve the identity of Fragilariforma species complex involving tropical species as $F$. brasiliensis, $F$. javanica, F. telum and F. strangulata. The environmental data showed in Table 1 indicate slightly acidic, oligo to mesotrophic environments.

After this analysis we consider that there are significant differences in morphometric parameters and ultrastructural analyses as spines, rimoportula and septum involving the taxonomy of this genus. We also propose $F$. amazonica as a new species, previously identified as $F$. nitzschioides var. brasiliensis.
The taxonomic synonymization presented above is in agreement with priority rules contained in the International Code of Nomenclature for Algae, Fungi, and Plants -Melbourne Code (McNeILl et al. 2012).

Regarding the morphometric analysis, our data corroborate the separation of F. amazonica, F. brasiliensis, F. strangulata and F. telum (Fig. 130), and these taxa should be recognized as distinct. Fragilariforma has a broad morphological variability and the analysis provides a more comprehensive approach to these characters. For a correct identification of species in Fragilariforma, populational analyses and measurement of environmental conditions in habitats where the species were collected should be prioritized. Our studies show that, despite the frequent occurrence of this genus in tropical environments, knowledge on its distribution and number of species is still uncertain. 


\section{ACKNowledgements}

This study was carried out within the framework of the AcquaSed project supported by funds from FAPESP (Fundação de Amparo à Pesquisa do Estado de São Paulo, AcquaSed Project, n ${ }^{\circ}$ 2009/538989 ), and was undertaken as part of PDA's PhD thesis (FAPESP doctoral fellowship 2014/13179-1 to PDA) at the Instituto de Botânica - IBt (São Paulo, Brazil). Funding was also provided by CNPq Conselho Nacional de Desenvolvimento Científico e Tecnológico (Grant 310940/2013-3 to DCB and 207702/2014-4-SWE to PDA) and LIST, Luxembourg Institute of Science and Technology (DIATOMS project). We deeply appreciate the valuable assistance of personnel from the agency in charge of the public water supply in São Paulo - SABESP/RHMS (Companhia de Saneamento do Estado de São Paulo, Divisão de Recursos Hídricos Metropolitanos Sudoeste). We thank Dr. Anton Igersheim (Naturhistorisches Museum Wien) for sending us information concerning Grunow's material. We also thank Dr. Jonathan Taylor for sharing Zambia material samples and Dr. Stéfano Zorzál for information about Paiva Castro reservoir. We thank all the students and technicians from the Department of Ecology, IBt, involved in field and laboratory work.

\section{REFERENCES}

Alfinito, S. \& Lange-Bertalot, H. (2013): Contribution to the knowledge of the freshwater algae of Sierra Leone (Tropical West Africa): diatoms from Loma Mountains and Bumbuna Falls, the Northern Province. - Biodiversity Journal 4: 135-178.

APHA - American Public Health Association (2005): Standard Methods for the Examination of Water and Wastewater, $21^{\text {st }}$ Edition, Washington, DC, $1368 \mathrm{pp}$.

Barber, H.G. \& Haworth, E.Y. (1981): A guide to the morphology of the diatom frustules with a key to the British freshwater genera. - Freshwater Biological Association, Scientific Publication 44: 1-111.

Brassac, N.M. \& LudwiG, T.A.V. (2003): Fragilariaceae (Bacillariophyceae) de rios da bacia do Iguaçu, Estado do Paraná, Brasil. - Revista Brasileira de Botânica 26: 311-318.

CARTER, J.R. \& DenNy, P. (1987): Freshwater algae of Sierra Leone IV. Bacillariophyceae; Part (II) diatoms from the coastal region of the southern province. - Nova Hedwigia 44: 229-275.

Cox, E.J. (1998): The identity and typification of some naviculoid diatoms (Bacillariophyta) from freshwater or brackish habitats. - Phycologia 37: 162-175.

Cox, E.J. (1999): Studies on the diatom genus Navicula Bory. VIII. Variation in valve morphology in relation to the generic diagnosis based on Navicula tripunctata (O.F. Müller) Bory. - Diatom Research 14: 207-237.

Dunck, B.; Nogueira, I.S. \& Machado, M.G. (2012): Planktonic diatoms in lotic and lentic environments in the Lago dos Tigres hydrologic system (Britânia, Goiás, Brazil): Coscinodiscophyceae and Fragilariophyceae. - Brazilian Journal of Botany 35: 181-193.

European Committee for Standardization (2003): Water Quality. - Guidance Standard for the Routine Sampling and Pretreatment of Benthic Diatoms from Rivers. European Standard EN 13946. - European Committee for Standardization, Brussels, $14 \mathrm{pp}$.

Ferrari, F. \& Ludwig, T.A.V. (2007): Coscinodiscophyceae, Fragilariophyceae e Bacillariophyceae (Achnanthales) dos rios Ivaí, São João e dos Patos, bacia hidrográfica do rio Ivaí, município de Prudentópolis, PR, Brasil. - Acta Botanica Brasilica 21: 421-441.
Gomes, D.F.; Caldas, O.; Da Silva, E.M.; Gell, P.A. \& WILLIAMS, D.M. (2012): Father Zimmermann (18711950): the first Brazilian diatomist. - Diatom Research 27: 177-188.

Hofmann , G.; Werum, M. \& Lange-Bertalot, H. (2011): Diatomeen im Süßwasser-Benthos von Mitteleuropa. Bestimmungsflora Kieselalgen für die ökologische Praxis. Über 700 der häufigsten Arten und ihre Ökologie. - 908 pp., A.R.G. Gantner, Ruggell.

Howard, K. \& Morales, E.A. (2012): Fragilariforma nitzschioides. - Diatoms of the United States. Available online 10/04/2015 at http://westerndiatoms.colorado. edu/taxa/species/fragilariforma_nitzschioides

Hustedt, F. (1949): Diatomeen von der Sinai-Halbinsel und aus dem Libanon-Gebiet. - Hydrobiologia 2: 24-55.

Index Nominum Algarum (2015): University Herbarium, University of California, Berkeley. - SiLvA, P. Available online 10/04/2015 at http://ucjeps.berkeley.edu/ $\mathrm{CPD} /$

JAHN, R. \& KusBer, W. (2009): A key to diatom nomenclature. - Diatom Research 24: 101-111.

Kilroy, C.; Sabbe, K.; Bergey, E.A.; Vyverman, W. \& Lowe, R. (2003): New species of Fragilariforma (Bacillariophyceae) from New Zealand and Australia. - New Zealand Journal of Botany 41: 535-554.

Kingston, J.C. \& Pappas, J.L. (2009): Quantitative shape analysis as a diagnostic and prescriptive tool in determining Fragilariforma (Bacillariophyta) taxon status. - Nova Hedwigia, Beiheft 135: 103-119.

Kingston, J.C.; Sherwood, A.R. \& Bengtsson, R. (2001): Morphology and taxonomy of several Fragilarifor$m a$ taxa from Fennoscandia and North America. - In: ECONOMOU-Amilli, A. (ed.): Proceedings of the 16th International Diatom Symposium, 25 Aug. - 1 Sept. 2000, Athens \& Aegean Islands. - pp. 73-88, Amvrosiou Press, Athens.

Krammer, K. \& Lange-Bertalot, H. (1991): Bacillariophyceae. 3. Teil: Centrales, Fragilariaceae, Eunotiaceae. - In: Ettl, H.; Gerloff, J.; Heynig, H. \& MollenHAUER, D. (eds): Süsswasserflora von Mitteleuropa, Vol. 2/3. - 576 pp., Gustav Fischer Verlag, Stuttgart - Jena.

LANDucci, M. \& Ludwig, T.A.V. (2005): Diatomáceas de rios da bacia hidrográfica Litorânea, PR, Brasil: Coscinodiscophyceae e Fragilariophyceae. - Acta Botanica Brasilica 19: 345-357.

McNeill, J.; Barrie, F.R.; Buck, W.R.; Demoulin, V.; Greuter, W.; Hawksworth, D.L.; Herendeen, P.S.; Knapp, S.; Marhold, K.; Prado, J.; Prud’Homme Van Reine, W.F.; Smith, G.F.; Wiersema, J.H. \& Turland, N.J. (2012): International Code of algae, fungi, and plants (Melbourne Code) adopted by the Eighteenth International Botanical Congress Melbourne, Australia, July 2011. - Regnum Vegetabile 154: $1-240$

Metzeltin, D. \& Lange-Bertalot, H. (1998): Tropical diatoms of South America I. About 700 predominantly rarely known or new taxa representative of the neotropical flora. - Iconographia Diatomologica 5: $1-695$.

Morales, E.A.; Siver, P. \& Trainor, F. (2001): Identification of diatoms (Bacillariophyceae) during ecological assessments: comparison between light microscopy and scanning electron microscopy techniques. - Proceedings of the Academy of Natural Sciences of 
Philadelphia 151: 95-103.

Morales, E.A.; Manoylov, K. \& Bahls, L.L. (2012): Fragilariforma horstii sp. nov. (Bacillariophyceae) a new araphid species from the northern United States of America. - Nova Hedwigia, Beiheft 141: 141-154.

Nardelli, M.S.; Bueno, N.C.; Ludwig, T.A.V; Tremarin, P.I. \& BARTOZEK, E.C.R. (2014): Coscinodiscophyceae and Fragilariophyceae (Diatomeae) in the Iguaçu River, Paraná, Brazil. - Acta Botanica Brasilica 28: 127-140.

Patrick, R.M. \& Reimer, C.W. (1966): The diatoms of the United States, exclusive of Alaska and Hawaii. Vol. 1: Fragilariaceae, Eunotiaceae, Achnanthaceae, Naviculaceae. - Monographs of the Academy of Natural Sciences of Philadelphia 13: 1-688.

R Core Team (2016): R: A language and environment for statistical computing. - R Foundation for Statistical Computing, Vienna, Austria. URL https://www.Rproject.org/.

Reid, G.; Huxley, R. \& Williams, D.M. (1994): Preliminary efforts towards a diatom type catalogue with an example from Fragilariforma virescens. - In: MARIANO, D. \& Montresor, M. (eds): Proceedings of the 13th International Diatom Symposium. - pp. 423-429, Biopress Ltd., Bristol.

RenBerg, I. (1977). Fragilaria lata, a new diatom species. Botaniser Notiser 130: 315-318.

Round, F.E.; Crawford, R.M. \& Mann, D.G. (1990): The Diatoms. Biology \& morphology of the genera. - 741 pp., Cambridge University Press, Cambridge.

Taylor, J.C.; Karthick, B.; Kociolek, J.P.; Wetzel, C.E. \& CocQuyt, C. (2014): Actinellopsis murphyi gen. et spec. nov.: A new small celled freshwater diatom (Bacillariophyta, Eunotiales) from Zambia. - Phytotaxa 178: 128-137.

Van Heurck, H. (1881): Synopsis des Diatomées de Belgique. Atlas. - 31-77 pls. Édité par l'auteur, Anvers.

VanLandingham, S.L. (1971): Catalogue of the fossil and recent genera and species of diatoms and their synonyms. Part IV. Fragilaria through Naunema: 1790 1791. - Cramer, Vaduz.

Wetzel, C.E. (2011): Biodiversidade e distribuição de diatomáceas (Ochrophyta, Bacillariophyceae) na bacia hidrográfica do Rio Negro, Amazonas, Brasil [PhD thesis]. - 1876 pp., Instituto de Botânica de São Paulo - Secretaria do Meio Ambiente.

Wetzel, C.E.; Lange-Bertalot, H.; Morales, E.A.; Bicudo, D.C.; Hoffmann, L. \& Ector, L. (2012a): Bicudoa amazonica gen. et sp. nov. (Bacillariophyta): a new freshwater diatom from the Amazon basin with a complete raphe loss in the Eunotioid lineage. - Phytotaxa 75 : $1-18$.

Wetzel, C.E.; Bicudo, D.C.; Ector, L.; Lobo, E.A.; Soininen, J.; Landeiro, V.L. \& BinI, L.M. (2012b): Distance decay of similarity in Neotropical diatom communities. - PLoS ONE 7: e45071.

Wetzel, C.E.; Morales, E.A.; Hinz, F.; Bicudo, D.C. \& EcTOR, L. (2013): Fragilariforma javanica comb. nov.: analysis of type material of a widely reported species with a tropical distribution. - Diatom Research 28: 373-379.

Williams, D.M. (1986): Comparative morphology of some species of Synedra Ehrenb. with a new definition of the genus. - Diatom Research 1: 131-152.

Williams, D.M. (1990): Fragilaria floridiana Hanna: ultra- structure of the valve and girdle and its transference to Fragilariforma Williams \& Round. - In: RiCARD, M. (ed.): Ouvrage dédié à la Mémoire du Professeur Henry Germain (1903-1989). - pp. 259-265. Koeltz Scientific Books, Königstein.

Williams, D.M. (1996): Notes on the genus Fragilariforma (Fragilariophyceae: Bacillariophyta) with a description of a new Miocene fossil species, Fragilariforma platensis. - Nova Hedwigia, Beiheft 112: 283-288.

WiLliams, D.M. (2001): Comments on the structure of "postauxospore' valves of Fragilariforma virescens. - In: JAHN, R.; Kociolek, J.P.; WitKowski, A. \& COMPère, P. (eds): Lange-Bertalot-Festschrift. Studies on diatoms. Dedicated to Prof. Dr. Dr. h.c. Horst LangeBertalot on the occasion of his 65th birthday. - pp. 103-117. A.R.G. Gantner, Ruggell.

Williams, D.M. \& Buczkó, K. (2016): Fragilariforma Hajósiae: re-description and revision of Pantocsek's species Diatoma fossile (Bacillariophyta). - Phytotaxa 244: 181-190.

Williams, D.M. \& Round, F.E. (1987): Revision of the genus Fragilaria. - Diatom Research 2: 267-288.

Williams, D.M. \& Round, F.E. (1988): Fragilariforma, nom. nov., a new generic name for Neofragilaria WiLLIAMS \& Round. - Diatom Research 3: 265-266.

WoJtal, A.Z. (2013): Species composition and distribution of diatom assemblages in spring waters from various geological formations in southern Poland. - Bibliotheca Diatomologica 59: 1-436.

Zanon, V. (1938): Diatomee della regione del Kivu (Congo Belga). - Commentationes, Pontificia Academia Scientiarum 2: 535-668.

Zimmermann, C.; Poulin, M. \& Pienitz, R. (2010): Diatoms of North America: The Pliocene-Pleistocene freshwater flora of Bylot Island, Nunavut, Canadian High Arctic. - Iconographia Diatomologica 21: 1-407.

ZimmermanN, C.S.J. (1919): Quelques diatomées nouvelles ou curieuses. - Broteria, Serie Botanica 17: 97-100.

(C) Czech Phycological Society (2017)

Received October 31, 2016

Accepted February 13, 2017 